\title{
Modelling eggshell maculation
}

\author{
Thomas W. Pike* \\ School of Life Sciences, University of Lincoln, Lincoln LN6 7DL, UK \\ *E-mail: tpike@lincoln.ac.uk
}

\begin{abstract}
The eggshells of many avian species are characterised by distinctive patterns of maculation, consisting of speckles, spots, blotches or streaks, the spatial-statistical properties of which vary considerably between (and often within) species. Understanding the mechanisms underlying the production of eggshell maculation would enable us to explore the costs and constraints on the evolution of maculation patterns, but as yet this area is surprisingly understudied. Here I present a simple model of eggshell maculation, which is based on the known biology of pigment deposition, and which can produce a range of realistic maculation patterns. In particular, it provides an explanation for previous observations of maculation heterogeneity and diversity, and allows testable predictions to be made regarding maculation patterns, including a possible signalling role.
\end{abstract}

Keywords: random field, pigmentation, protoporphyrin, shell-gland membrane

\section{INTRODUCTION}

The great diversity of avian eggshell pigmentation and its possible adaptive significance has fascinated biologists for over a century (Poulton, 1890). Ancestrally, avian eggshells were most likely homogenously white and immaculate (Wallace, 1889; Kilner, 2006), although not necessarily devoid of pigment (Kennedy and Vevers, 1975). Since then, however, they have evolved remarkable variation in both the basal ground colour and in the presence and patterns of superficial pigmentation, or maculation, which can include speckles, spots, blotches and streaks (Kilner, 2006; see illustrative examples in Cassey et al., 2011; Brulez et al., 2015 and references therein; Figure 1). A number of hypotheses have been proposed to explain the functional significance of eggshell maculation (reviewed in Underwood and Sealy, 2002; Kilner, 2006; Reynolds et al., 2009; Maurer et al., 2011; Brulez et al., 2015). These include, but are not limited to, crypsis to avoid predation (e.g. Götmark, 1993) and brood parasitism (e.g. Davies and Brooke, 1989), egg recognition (e.g. Pike, 2011), thermoregulation (e.g. Bakken et al., 1978), signalling maternal health (De Coster et al., 2012), increasing eggshell strength (Gosler et al., 2005), and providing defence against bacterial infection (Ishikawa et al., 2010). However, despite renewed interest in the function of eggshell maculation (Brulez et al., 2015), we still have a relatively poor understanding of the underlying causal mechanisms, even though this is crucial to understanding the evolution of maculation patterns and the factors constraining their production.

The various layers of the avian eggshell are formed sequentially as the immature egg rotates within the shell gland (or uterus) (Weiner and Addadi, 1991; Lavelin et al., 2000). Eggshell formation ceases after the deposition of a surface crystal layer, and is completed by the formation of a thin cuticular layer of eggshell accessory material (Board and Sparks, 1991). Eggshell pigments are deposited during the latter stages of eggshell formation and so typically occur either within the upper layers of the eggshell or superficially on the eggshell's surface (Poole, 1965; Breen and De Bruyn, 1969; Soh et al., 1993; Wang et al., 2007; (a)

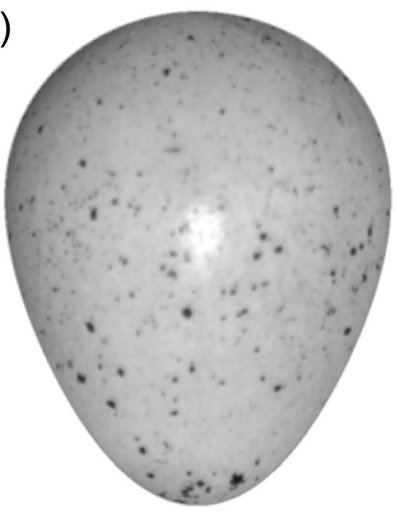

(b)

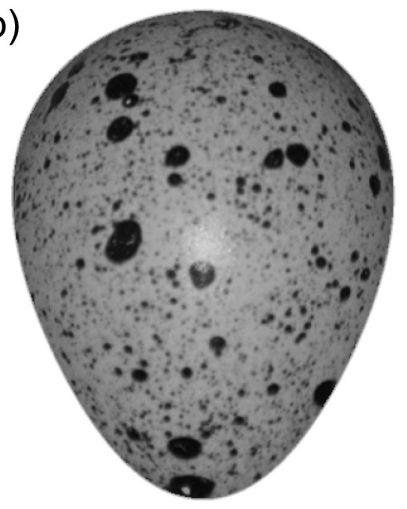

(c)

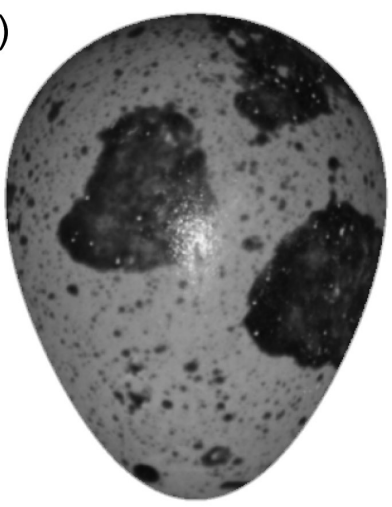

(d)

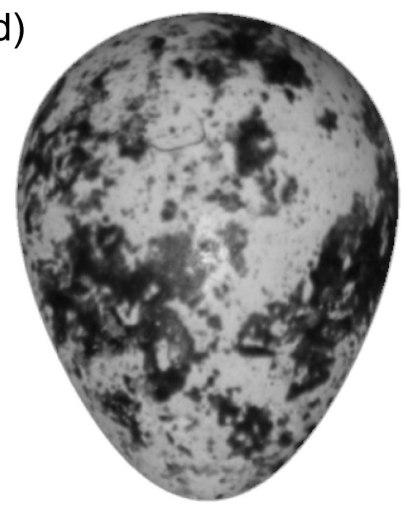

Figure 1 (a-d) Japanese quail (Coturnix japonica) eggs showing examples of different maculation patterns, varying in spot size, distribution and 'smoothness'. 
Samiullah and Roberts 2013). There are two main types of pigments responsible for the colouration and patterning on eggshells: biliverdin IX $\alpha$ produces a blue-green hue, while protoporphyrin IX produces brownish hues (Kennedy and Vevers, 1975). Homogeneously deposited pigment is responsible for the underlying base colour of the egg, while distinct patterns of maculation result from the heterogeneous deposition of relatively large amounts of pigment, typically protoporphyrin IX (Sparks, 2011). During the production of maculation, pigment granules in the apical cells of the shell gland epithelium (Tamura and Fujii, 1966; Poole, 1967) are secreted into the lumen shortly before oviposition (Poole, 1965; Tanaka et al., 1977; Soh et al., 1989; Tamura and Fujii, 1966) where they gather to form pigment masses between folds of the mucous membrane lining the shell gland (Soh et al., 1989). Contraction of the shell gland membrane then deposits these accumulated pigments onto the eggshell's surface (Soh et al., 1993), resulting in the speckles, spots, blotches and streaks, if the egg is in rotation (Solomon, 1987; Sparks, 2011), characteristic of the particular species or individual.

The aim of this paper is to present a simple model of eggshell maculation that is consistent with our biological understanding of the process, and which can account for the majority of maculation patterns observed in the eggs of real birds. Specifically, the model is based on the known morphology and functionality of the shell gland membrane, and explores the effects of varying each of the different stages (from membrane structure through to pigment accumulation and deposition) on the generation of maculation patterns. It is hoped that this will provide a foundation for future work on the evolution and function of maculation, by allowing the construction of models to generate maculation patterns for further theoretical or empirical exploration, and in the development of testable hypotheses relating to the costs and constraints of maculation production.

\section{MODEL}

\subsection{Overview}

I start with a model of the shell gland membrane, consisting of a continuous spherical surface comprising a 'landscape' of peaks and troughs with defined spatial-statistical properties (Figure 2a). It is assumed that this membrane secretes pigment, which accumulates in the troughs of the folds before being transferred to the eggshell surface during contraction of the shell gland membrane. These latter two processes are simply modelled by 'filling up' the troughs in the membrane with pigment to a predefined relative height (Figure $2 \mathrm{~b}$ ), quantising the membrane into pigmented and unpigmented regions (Figure 2c), and wrapping this quantised membrane around a sphere, which is then reshaped to resemble an egg (Figure $2 d$ ).
Variation in the spatial-statistical properties of the membrane (e.g. the depth, size and/or number of troughs) and/or the characteristics of the pigment filling the troughs in the membrane can produce a wide range of maculation patterns encompassing a large majority of the variation observed in real birds' eggs. These are explored in more detail below.

\subsection{Varying the spatial-statistical properties of the shell gland membrane}

The shell gland membrane is modelled as a spatially correlated (spherical) random field (i.e. a continuous spherical surface with a known spatial correlation structure) using unconditional Gaussian simulation, a common procedure in geostatistics (Diggle and Ribeiro, 2006). The particular, spatial-statistical properties of this random field are defined by an associated semivariogram model, which describes the average squared difference in membrane surface height between two given points as a function of the distance between those points (Olea, 1999). Semivariograms are widely used in geostatistics and there are a large number of texts dealing with their construction and interpretation, and readers are referred there for further information (e.g. see Olea, 1999 for an accessible introduction to the topic). Of particular importance here is that semivariogram functions typically show an initial increase with distance (because the surface heights at two spatially proximate locations tend to be more similar than those at two distant locations) before asymptoting at a distance, known as the range ( $r$ [where $0 \leq r \leq 180$ ], measured here in degrees), after which the heights at any given spatial locations cease to be correlated (Figures 3a and 4a). This relationship can be approximated using a variety of functions, of which the Gaussian and exponential functions were found to be useful for producing eggshell maculation patterns. Gaussian functions produce membranes with a smooth transition between peaks and troughs, and hence result in 'rounded' regions of maculation (Figure 3a; see also Figure 1b); in contrast, exponential functions result in 'jagged' areas of maculation (Figure 3a; see also Figure 1d). By varying the value of $r$ it is possible to control the degree of spatial correlation: specifically, increasing the value of $r$ (i.e., the distance at which the function asymptotes) results in random fields with increasingly 'coarse' autocorrelation, resulting in wider spaced and larger regions of pigmentation (Figure 3b; see also Figure 1a,b,c).

A further characteristic of many species' eggs is the skewed distribution of maculation along the long axis of the egg, such that maculation occurs predominantly at one pole or the other (e.g. see Figure 1 in Gosler et al., 2000 for examples in great tit [Parus major] eggs). This can be explicitly modelled by including a linear trend along one axis of the random field (specifically by varying the value of the trend coefficient, $\beta$; Olea, 1999), and can therefore 


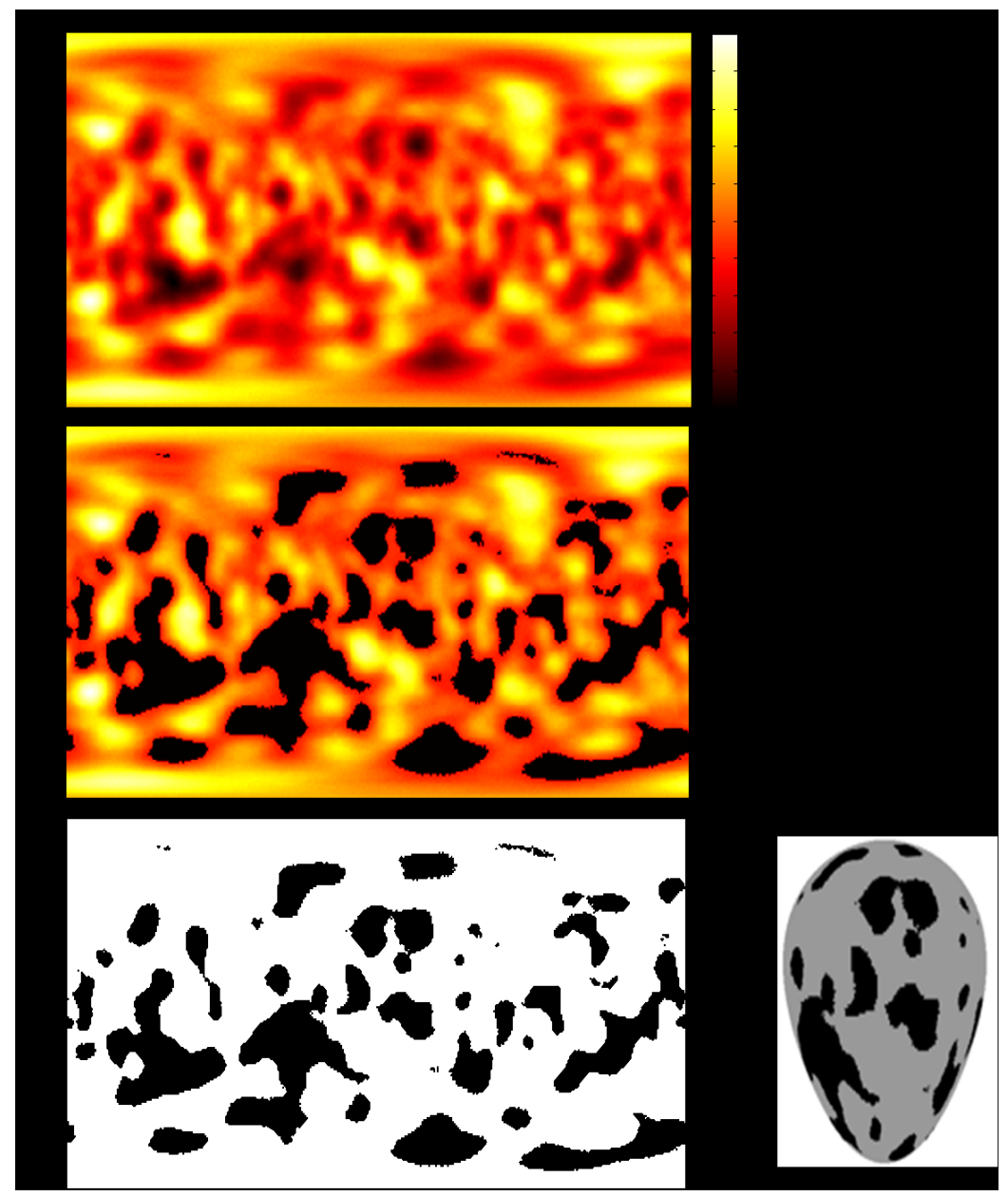

Figure 2 Modelling eggshell maculation. (a) The shell gland membrane is modelled as a spherical random field, here 'spread out' and represented by its equirectangular projection, with colours denoting the relative elevation of peaks and troughs among folds in the membrane. The membrane is simulated with a spatial resolution of 1 degree. (b) The membrane showing pigment (homogeneous black regions) partially filling the troughs. (c) The quantised membrane showing pigmented and non-pigmented regions. (d) The quantised pigment pattern mapped onto a three-dimensional model of an egg, following 'contraction' of the shell gland membrane. I assumed the egg had an underlying base colour (ranging from black [0] to white [1]), here set to 0.6. The shape of the egg was calculated following Todd and Smart (1984) assuming the following shape coefficients: $C_{1}=0.7, C_{2}=0.2, C_{3}=0.0$ and $c_{4}=-0.05$, and a height to breadth ratio of 1.3. In this example, the membrane was modelled using the following parameters: Gaussian semivariogram function; $r=10 ; q=0.4 ; d=1 ; \beta=0$.

simulate maculation with varying degrees of polarisation: when $\beta=0$, there is no trend and maculation is distributed evenly along the long axis of the egg; increasing values of $\beta$ result in increasingly strong trends, and hence increasingly polarised maculation (Figure $4 a, b)$.

By combining random fields with different spatialstatistical properties it is possible to create more complex membrane configurations, for example combining two or more patterns with differing spatial-statistical properties (e.g. spots combined with larger patches of pigmentation; Figure $4 j, I)$.

\subsection{Varying pigment deposition}

I consider that pigment can vary in both quantity $(q$, as a proportion of overall membrane height, from a complete absence of pigment $[q=0]$ to where the membrane is filled with pigment to the top of the highest peak $[q=1]$ ) and optical density $(d$, from transparent $[d=0]$ to opaque $[d=1])$. Varying $q$ affects how deeply the troughs in the membrane are filled, allowing membranes with the same spatial-statistical structure to produce very different patterns of maculation (Figure $4 \mathrm{c}, \mathrm{d}, \mathrm{e}, \mathrm{f})$. Varying $d$ can produce maculation that is heterogeneously coloured, with areas of both heavy and weak pigmentation: when $0<d<1$ deeper troughs, containing more pigment, will produce darker regions of maculation than shallower troughs; similarly, deeper (typically central) regions of troughs will produce darker maculation than shallower (typically peripheral) regions (Figure $4 \mathrm{~g}$; see also Figure 1c,d).

By 'rotating' the egg during or shortly after the deposition of the pigment it is possible to simulate the effects of streaking (Figure 4h), and by applying pigment to the eggshell more than once (for example before and 
(a)

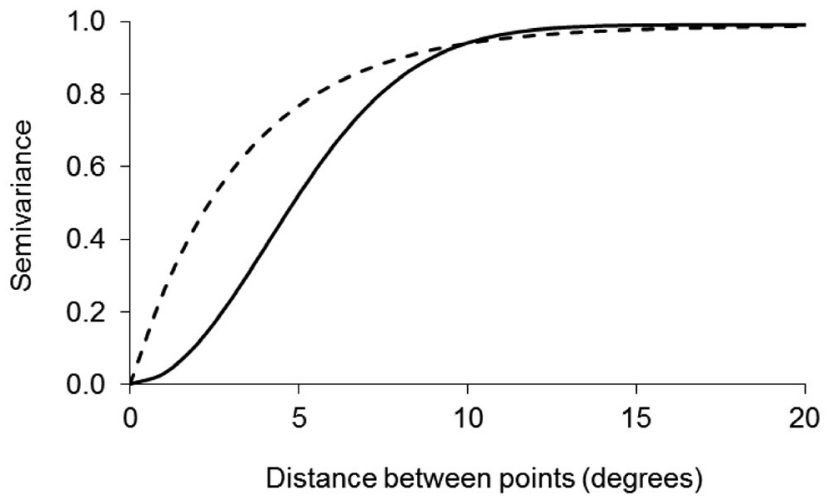

(c)

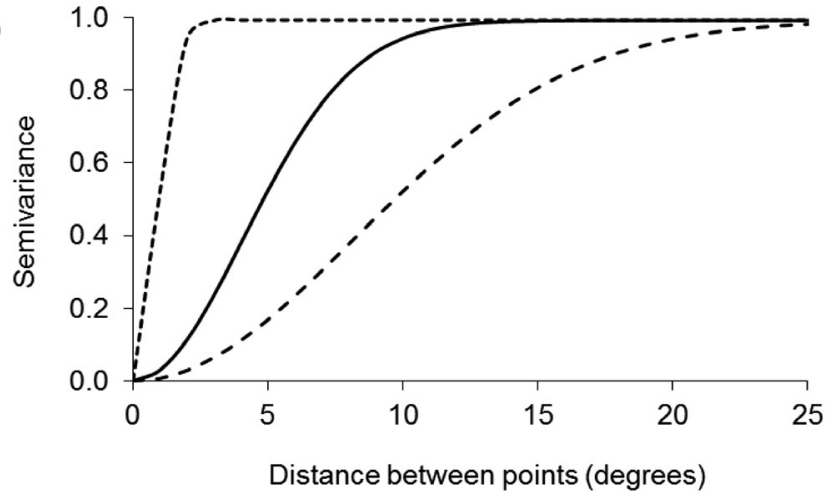

(b)

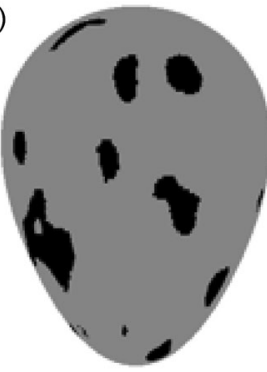

Gaussian

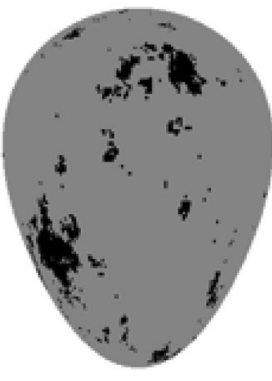

Exponential (d)

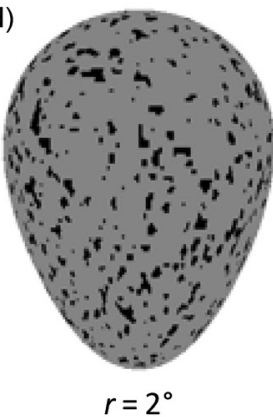

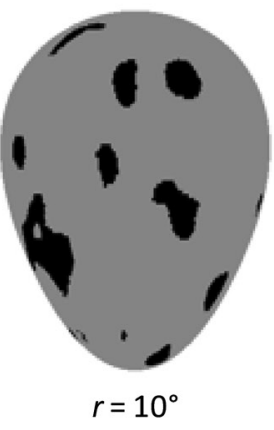

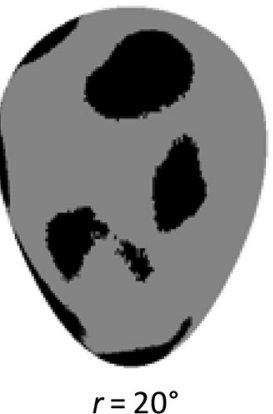

Figure 3 The effect of varying the type and range parameter $(r)$ of the semivariogram function on eggshell maculation. The semivariogram function models the average (squared) difference of the membrane surface values between two given points (the semivariance) as a function of the distance between those points, and hence defines the spatial-statistical properties of the eggshell membrane. (a) Gaussian (solid line) and exponential (dashed line) semivariogram functions, both with a fixed $r=10^{\circ}$, and (b) representative realisations of resulting maculation patterns. (c) Gaussian semivariogram functions with $r=2^{\circ}$ (dotted line), $r=10^{\circ}$ (solid line) and $r=20^{\circ}$ (dashed line) (note that for both Gaussian and exponential semivariograms, $r$ is defined as the distance at which the semivariance first reaches $95 \%$ of the asymptotic height; Olea, 1999), and (d) representative realisations of resulting maculation patterns. In all cases the membrane was modelled using the following additional parameters: $q=0.4 ; d=1 ; \beta=0$, using a semivariogram with a fixed asymptotic value (known as the sill) and y-axis intercept (nugget) (sensu Olea, 1999).

after rotation of the egg) it is possible to generate complex patterns, for instance, simulating maculation within a distinct eggshell layer combined with superficial eggshellsurface maculation (Figure 4i) or combining spots and streaks (Figure $4 \mathrm{k}$ ).

\subsection{Implementation}

The model was implemented in Matlab (Mathworks, Natick, MA) with calls to the 'predict.gstat' function in the gstat package (Pebesma, 2004) for $R$ (version 2.15.2) to generate the spherical random fields. All code is available on request from the author.

\section{DISCUSSION}

Here I present a simple model of eggshell maculation, which is based on the biological mechanisms underlying the process of pigment deposition. Specifically, by varying the spatial-statistical properties of the folds in the shell gland membrane, along with the amount of pigment secreted by the membrane epithelium, the model can simulate the majority of maculation patterns that have been observed in real birds' eggs (e.g. Brulez et al., 2015) and can produce patterns that are statistically indistinguishable from those generated naturally. For example, there is no statistical difference between the maculation patterns present on real Japanese quail eggs (from Pike, 2011) and simulated patterns that appear, at least to human observers, to provide good species-typical matches (Figure 5), strongly suggesting that the model generates biologically plausible maculation patterns. Differences in membrane characteristics between species may therefore account for much of the inter- and intraspecific variation in maculation patterns observed, while also explaining phenomena such as within clutch consistency in maculation patterns, the heritability of maculation, and observed links between maculation and the laying female's health and physiological status. 
(a)

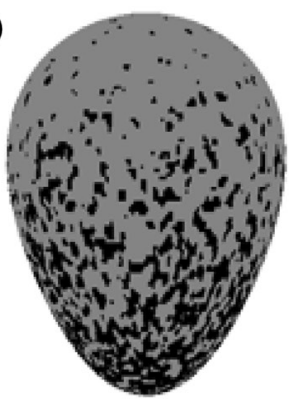

(e)

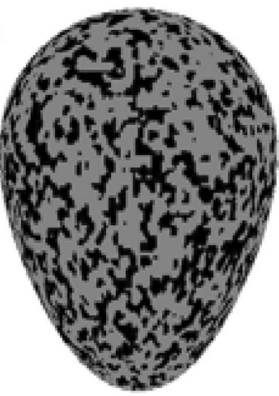

(i)

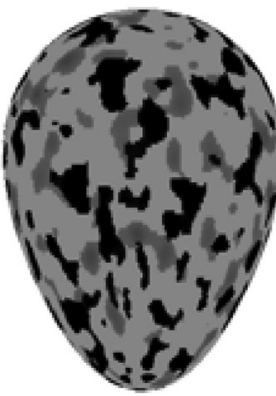

(b)

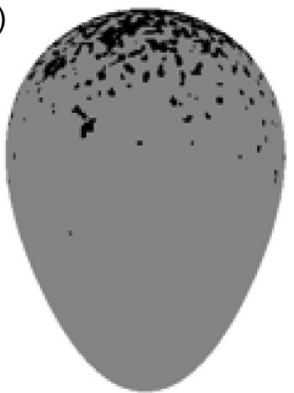

(f)

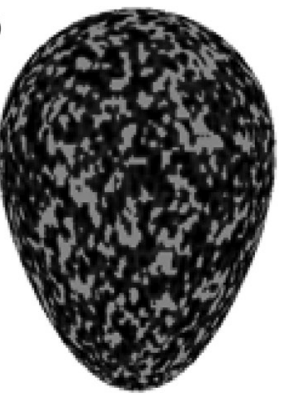

(j)

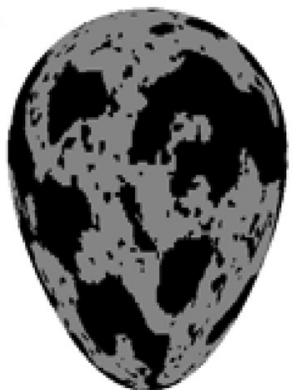

(c)

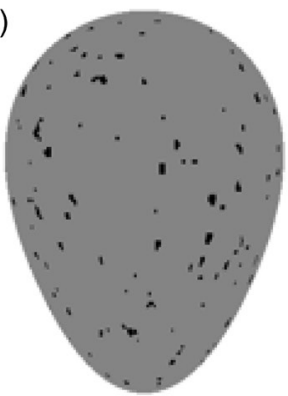

(g)

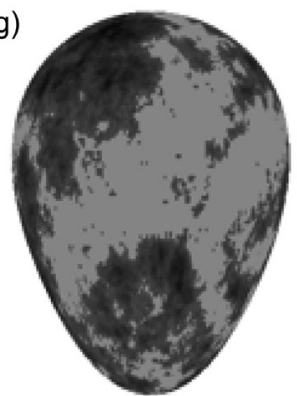

(k)

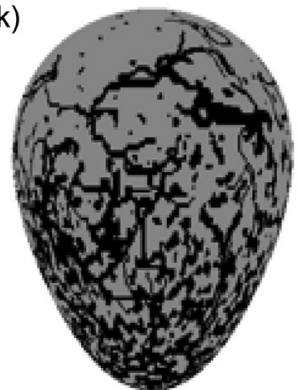

(d)

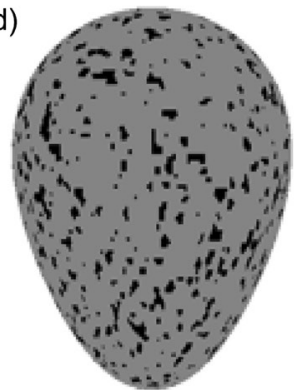

(h)

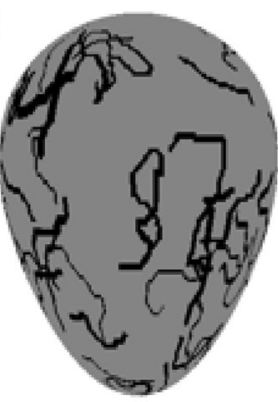

(I)

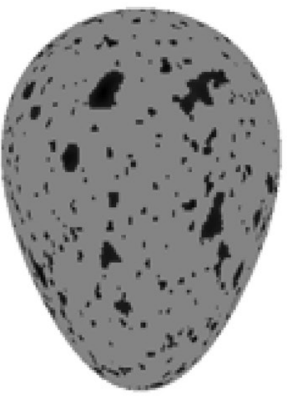

Figure 4 Examples of simulated maculation patterns. Please refer to the text for full details. Maculation was modelled using the following parameters: (a) Gaussian semivariogram function; $r=2^{\circ} ; q=0.4 ; d=1 ; \beta=0.02$; (b) Gaussian semivariogram function; $r=2^{\circ} ; q=0.4 ; d=1 ; \beta=0.05$ (and the resulting membrane flipped along the long axis of the egg); (c) Gaussian semivariogram function; $r=2^{\circ} ; q=0.3 ; d=1 ; \beta=0 ;(d)$ Gaussian semivariogram function; $r=2{ }^{\circ} ; q=0.4 ; d=1 ; \beta=0 ;$ (e) Gaussian semivariogram function; $r=2^{\circ} ; q=0.5 ; d=1 ; \beta=0 ;$ (f) Gaussian semivariogram function; $r=2^{\circ} ; q=0.6 ; d=1 ; \beta=0 ;$ (g) exponential semivariogram function; $r=10^{\circ} ; q=0.6 ; d=0.6 ; \beta=0$; (h) Gaussian semivariogram function; $r=2^{\circ} ; q=0.3 ; d=1 ; \beta=0 ;$ streaking was simulated by 'rotating' the egg around a static random field according to a correlated random walk with equal step lengths of $1^{\circ}$ and directions chosen from a uniform distribution on $\left[-20^{\circ}, 2^{\circ}\right]$; (i) Gaussian semivariogram function; $r=5^{\circ} ; q=0.4 ; d=0.3$ and $1 ; \beta=0$; pigment was applied twice, with a $90^{\circ}$ rotation between applications; (j) Gaussian semivariogram function; $r=2^{\circ}$ and $10^{\circ} ; q=0.4 ; d=1$; $\beta=0$; two membranes with different spatial-statistical properties were combined by overlaying them and retaining the lowest value at each spatial location; (k) Gaussian semivariogram function; $r=2^{\circ} ; q=0.4$ and $0.2 ; d=1 ; \beta=0.02$; pigment was applied twice, with rotation and streaking on the second application; (I) Gaussian semivariogram function; $r=2^{\circ}$ and $5^{\circ} ; q=0.4 ; d=1 ; \beta=0$; two membranes combined as in (j).

Although there are marked between-species differences in maculation patterns, many species of birds have evolved within-clutch uniformity as well as individual distinctiveness in maculation in order to aid distinguishing between an individual's own eggs and those of a conspecific or a brood parasite (Baker, 1913; Davies and Brooke, 1989; Cherry et al., 2007; Pike, 2011). Japanese quail, for instance, exhibit remarkably low within-clutch variation in the spatial-statistical properties of maculation patterns in their eggs compared with the variation between females (Pike, 2011). Assuming that pigmentation patterns are determined by the spatial-statistical properties of the shell gland membrane, consecutive eggs in a clutch would be exposed to the same membrane, albeit with small conformational changes resulting from the precise position of the egg within the gland, or slight variation in the strength and distribution of uterine contractions, and so would be expected to exhibit maculation with similar (but not identical) spatial-statistical properties. This is certainly consistent with previous observations (e.g. Pike, 2011), although to my knowledge has not been addressed experimentally. This potential lack of flexibility in the ability to physiologically control the gross pigmentation of their eggs may explain why some species, such as Japanese quail, have evolved behavioural strategies to minimise the conspicuous of their eggs (Lovell et al., 2013). 


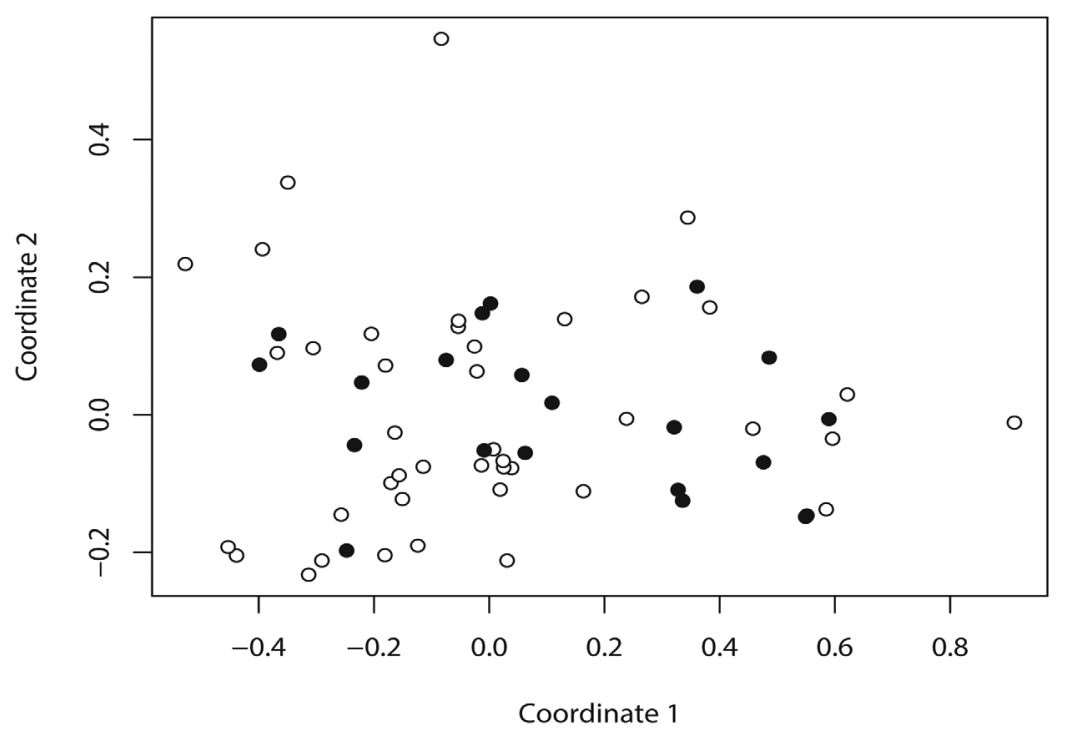

Figure 5 Non-metric multidimensional scaling (MDS) plot showing the locations of the 42 Japanese quail eggs described by Pike (2011) (white points) and 20 eggs simulated using the model described here (black points) in 'maculation-space' (i.e., a multidimensional phenotypic space describing various statistical properties of eggshell maculation patterns, as described in detail in Pike (2011)). The locations of the two sets of data points did not differ significantly (non-parametric MANOVA: $F_{1,61}=1.43, P=0.229$ ).

Eggshell maculation has been shown to be genetically female sex-linked in great tits, being inherited from mother to daughter (Gosler et al., 2000; but see Mahler et al., 2008), although as is the case for within-clutch uniformity the mechanism of maculation heritability has never been explored. If there was a genetic component to the spatialstatistical structure of the shell gland membrane, this could explain the heritability of eggshell pigmentation patterns.

The model presented here also allows us to make predictions linking maculation patterns to variation in the condition or physiology of the laying female. In humans, characteristics of the mucous membrane lining the respiratory tract (which shares many similarities with the avian shell gland membrane; Sevoian and Levine, 1957), such as membrane flexibility and the extent of folding, are known to be affected by certain diseases (Wiggs et al., 1997), while respiratory diseases in birds have been shown to affect the shell gland and the deposition of pigment (Sevoian and Levine, 1957). It could therefore be predicted that condition- or health-related variation in the shell gland membrane impacts on the maculation patterns produced. Indeed, in various species body condition and health parameters are known to correlate with eggshell maculation (Martínez-de la Puente et al., 2007; Sanz and Garcia-Navas, 2009; Duval et al., 2014), although why this should be has not been explored. It is also noteworthy that certain characteristics of the shell gland membrane (Olson et al., 1978) and the production of pigment (Soh and Koga, 1994) are known to be under hormonal control, which may provide a mechanistic link between the physiological status of a laying female and the maculation of her eggs. If an empirical link can be demonstrated between eggshell maculation, condition or health, and the underlying mechanism of maculation production, this would provide evidence for a possible signalling role of maculation patterns (De Coster et al., 2012).

In summary, the simple model of eggshell maculation presented here, which is based on the known biology of pigment deposition and which can produce a range of realistic maculation patterns, provides an explanation for previous observations of maculation heterogeneity and diversity, and allows testable predictions to be made regarding maculation patterns, including a possible signalling role.

\section{ACKNOWLEDGMENTS}

Thank you to Charles Deeming and Oliver Burman for valuable discussions during the production of this paper, and to Daniel Hanley for insightful and supportive comments on an earlier version.

\section{REFERENCES}

Bakken, G.S., Vanderbilt, V.C., Buttemer, W.A. and Dawson, W.R. (1978) Avian eggs: thermoregulatory value of very high nearinfrared reflectance. Science, 200, 321-323.

Board, R.G. and Sparks, N.H.C. (1991) Shell structure and formation in avian eggs. In: Deeming D.C. and Ferguson M.W.J. (eds), Egg Incubation: its Effects on Embryonic Development in Birds and Reptiles, pp. 71-86. Cambridge University Press, Cambridge.

Breen, P.C. and De Bruyn, P.P.H. (1969) The fine structure of the secretory cells of the uterus (shell gland) of the chicken. J. Morphol., 128, 35-66.

Brulez, K., Pike, T.W. and Reynolds, S.J. (2015) Egg signalling: the use of visual, auditory and chemical stimuli. In: Deeming, D.C. and Reynolds, S.J. (eds), Nests, Eggs, and Incubation: New Ideas about Avian Reproduction. Oxford University Press, Oxford. 
Cherry, M.I., Bennett, A.T.D. and Moskat, C. (2007) Host intra-clutch variation, cuckoo egg matching and egg rejection by great reed warblers. Naturwissenschaften, 94, 441-447.

Davies, N.B. and Brooke, M.D.L. (1989) An experimental study of co-evolution between the cuckoo, Cuculus canorus, and its hosts. I. Host egg discrimination. J. Anim. Ecol., 58, 207-224.

De Coster, G.1., De Neve, L. and Lens, L. (2012) Intraclutch variation in avian eggshell pigmentation: the anaemia hypothesis. Oecologia, 170, 297-304.

Diggle, P.J. and Ribeiro, P.J. (2006) Model-based geostatistics. Springer, New York.

Duval, C., Zimmer, C., Mikšík, I., Cassey, P. and Spencer, K.A. (2014) Early life stress shapes female reproductive strategy through eggshell pigmentation in Japanese quail. Gen. Comp. Endocrinol., 208, 146-53.

Cassey, P., Maurer, G., Lovell, P.G. and Hanley, D. (2011) Conspicuous eggs and colourful hypotheses: testing the role of multiple influences on avian eggshell appearance. Avian Biol. Res., 4, 185-195.

Gosler, A.G., Barnett, P.R. and Reynolds, S.J. (2000) Inheritance and variation in eggshell patterning in the great tit Parus major. Proc. R. Soc. B, 267, 2469-2473.

Gosler, A.G., Higham, J.P. and Reynolds, S.J. (2005) Why are birds' eggs speckled? Ecol. Lett., 8, 1105-1113.

Götmark, F. (1993) Conspicuous coloration in male birds: favoured by predation in some species, disfavoured in others. Proc. $R$. Soc. B, 253, 143-146.

Ishikawa, S., Suzuki, K., Fukuda, E., Arihara, K., Yamamoto, Y., Mukai, T. and Itoh, M. (2010) Photodynamic antimicrobial activity of avian eggshell pigments. FEBS Lett., 584, 770-774.

Kennedy, G.Y. and Ververs, H.G. (1975) A survey of avian eggshell pigments. Comp. Biochem. Physiol., 55, 117-123.

Kilner, R.M. (2006) The evolution of egg colour and patterning in birds. Biol. Rev., 81, 383-406.

Lavelin, I., Meiri, N. and Pines, M. (2000) New insight in eggshell formation. Poultry Sci., 79, 1014-1017.

Lovell, P. George, Ruxton, G.D., Langridge, K.V. and Spencer, K.A. (2013) Egg-laying substrate selection for optimal camouflage by quail. Curr. Biol., 23, 260-264.

Mahler, B., Confalonieri, V.A., Lovette, I.J. and Reboreda, J.C. (2008) Eggshell spotting in brood parasitic shiny cowbirds (Molothrus bonariensis) is not linked to the female sex chromosome. Behav. Ecol. Sociobiol., 62, 1193-1199.

Martínez-De La Puente, J., Merino, S., Moreno, J., Tomas, G., Morales, J., Lobato, E., García-Fraile, S. and Martínez, J. (2007) Are eggshell spottiness and colour indicators of health and condition in blue tits Cyanistes caeruleus? J. Avian Biol., 38, 377-384.

Maurer, G., Portugal, S.J. and Cassey, P. (2011) An embryo's eye view of avian eggshell pigmentation. J. Avian Biol., 42, 494-504.

Olea, R.A. (1999) Geostatistics for engineers and earth scientists. Kluwer Academic Publishers, Dordrecht.

Olson, D.M., Biellier, H.V. and Hertelendy, F. (1978) Shell gland responsiveness to prostaglandins $\mathrm{F} 2 \alpha$ and $\mathrm{E} 1$ and to arginine vasotocin during the laying cycle of the domestic hen (Gallus domesticus). Gen. Comp. Endocrinol., 36, 559-565.

Pebesma, E.J. (2004) Multivariable geostatistics in S: the gstat package. Comp. Geosci., 30, 683-691.

Pike, T.W. (2011) Egg recognition Japanese in quail. Avian Biol. Res., 4, 231-236.
Poole, H.K. (1965) Spectrophotometric identification of eggshell pigments and timing of superficial pigment deposition in Japanese quail. Proc. Soc. Exp. Biol. Med., 119, 547-551.

Poole, H.K. (1967) A microscopic study of uterine eggshell pigment in Japanese quail. J. Hered., 58, 200-203.

Poulton, E.B. (1890) The Colours of Animals: Their Meaning and Use. D. Appleton and Co., New York.

Reynolds, S.J., Martin, G.R. and Cassey, P. (2009) Is sexual selection blurring the functional significance of eggshell coloration hypotheses? Anim. Behav., 78, 209-215.

Sanz, J.J. and García-Navas, V. (2009) Eggshell pigmentation pattern in relation to breeding performance of blue tits Cyanistes caeruleus. J. Anim. Ecol., 78, 31-41.

Samiullah, S. and Roberts, J.R. (2013) The location of protoporphyrin in the eggshell of brown-shelled eggs. Poultry Sci., 92, 2783-2788.

Sevoian. M. and Levine, P.P. (1957) Effects of infectious bronchitis on the reproductive tracts, egg production, and egg quality of laying chickens. Avian Dis., 1, 136-164.

Soh, T., Koga, O. and Tanaka, K. (1989) Involvement of ovulation mechanism(s) in the accumulation of pigment in the shell gland of the Japanese quail (Coturnix coturnix japonica). Poultry Sci., 68, 1156-1158.

Soh, T., Fujihara, N. and Koga, O. (1993) Observations of pigment accumulation in the epithelium of the shell gland and superficial pigmentation on the egg shell in Japanese quail. J. Fac. Agr. Kyushu Univ., 38, 73-80.

Soh, T. and Koga O. (1994) The effects of sex steroid hormones on the pigment accumulation in the shell gland of Japanese quail. Poultry Sci., 73, 179-185.

Solomon, S.E. (1987) Egg shell pigmentation. In: Wells, R.G. and Belyarin, C.G. (eds), Egg quality: current problems and recent advances, pp. 147-157. Butterworths, London.

Sparks, N.H.C. (2011) Eggshell pigments - from formation to deposition. Avian Biol. Res., 4, 162-167.

Tamura, T. and Fujii, S. (1966) Histological observations on the quail oviduct; on the secretions in the mucous epithelium of the uterus. J. Fac. Fish. Anim. Husb. Hiroshima Univ., 6, 357-371.

Tanaka, K., Imai, T. and Koga, O. (1977) Superficial pigmentation of egg shell in Japanese Quail, Coturnix coturnix japonica. Jpn. Poult. Sci., 14, 229-231.

Todd, P.H. and Smart, I.H.M. (1984) The shape of birds' eggs. J. Theor. Biol., 106, 239-243.

Underwood, T.J. and Sealy, S.G. (2002) Adaptive significance of egg colouration. In: Deeming, D.C. (ed.), Avian Incubation: Behaviour, Environment and Evolution, pp. 280-289. Oxford University Press, Oxford.

Wang, X.-T., Deng, X.-M., Zhao, C.-J., Li, J.-Y., Xu, G.-Y., Lian, L.-S. and Wu, C.-X. (2007) Study of the deposition process of eggshell pigments using an improved dissolution method. Poultry Sci., 86, 2236-2238.

Wallace, A.R. (1889) Darwinism: an exposition of the theory of natural selection, with some of its applications. MacMillan, New York.

Weiner, S. and Addadi, L. (1991) Acidic macromolecules of mineralized tissues: The controllers of crystal formation. Trends Biochem. Sci., 16, 52-256.

Wiggs, B.R., Hrousis, C.A., Drazen, J.M. and Kamm, R.D. (1997) On the mechanism of mucosal folding in normal and asthmatic airways. J. Appl. Physiol., 83, 1814-21. 